\title{
Neighborhoods of a Compact Non-Singular Algebraic Curve Imbedded in a 2-Dimensional Complex Manifold
}

\author{
By \\ Osamu SUZUKI*
}

\section{Introduction}

Let $C$ be a compact non-singular algebraic curve imbedded in a 2-dimensional complex manifold $S$. In this paper we consider the following problem:

Under what conditions does there exist a non-constant holomorphic function defined on a small neighborhood of $C$ ?

The signature of the normal bundle $N_{C}$ is not sufficient to solve our problem (see, Table in $\S 6$ and Theorem 2 in $\S 5$ ). Hence we have to introduce the concept of a regularly half pseudoconvex neighborhood system of $C$ (for definition, see (1.1)). Then the necessary and sufficient condition is given in the following

Main Theorem. There exists a non-constant holomorphic function defined on a neighborhood of $C$ if and only if either (1) $N_{C}<0$ or (2) $N_{C}$ is of finite order (for difinition, see $\S 1$ ) and $C$ has a regularly half pseudoconvex neighborhood system.

Therefore we can conclude that our problem is completely solved by using the normal bundle and pseudoconvexity of a neighborhood system of $C$. Detailed results will be summarized in Table in $\S 6$.

The author would like to express his gratitude to Professor S. Iitaka for his encouragement during the preparation of the present paper.

Communicated by S. Nakano, September 19, 1974.

* Graduate School, University of Tokyo, Tokyo. 


\section{$\S 1$. Notations}

First we recall terminology on complex line bundles. Let $E$ be a complex line bundle on $S$ which is expressed as $E=\left\{f_{\lambda \mu}\right\}$ with respect to some open covering $\left\{U_{\lambda}\right\}$ of $S . \quad \mathcal{O}(E)$ (resp. $\mathscr{E}(E)$ ) denotes the sheaf of germs of holomorphic (resp. $C^{\infty}$-differentiable) sections of $E$. A metric $\left\{a_{\lambda}\right\}$ of $E$ is a system of positive $C^{\infty}$-functions $a_{\lambda}$ on $U_{\lambda}$ satisfying

$$
a_{\mu}=\left|f_{\lambda \mu}\right|^{2} a_{\lambda} \quad \text { on } \quad U_{\lambda} \cap U_{\mu} \neq \phi .
$$

$E$ is called positive if there exists a metric $\left\{a_{\lambda}\right\}$ such that the hermitian matrix $\left(A_{\alpha \bar{\beta}}^{\lambda}\right)$ defined by

$$
-\partial \bar{\partial} \log a_{\lambda}=\sum_{\alpha, \beta=1}^{2} A_{\alpha \bar{\beta}}^{\lambda} d z_{\lambda}^{\alpha} \wedge d \bar{z}_{\beta}^{\lambda} \quad \text { on each } U_{\lambda}
$$

is positive definite where $\left(z_{\lambda}^{1}, z_{\lambda}^{2}\right)$ denotes a system of local coordinates. In the case where $E$ is topologically trivial line bundle, $E$ is called of finite order if there exists a positive integer $k$ such that $E^{k}=E \otimes E$ $\otimes \cdots \otimes E$ ( $k$-times tensor product) is analytically trivial. If not, it is called of infinite order. A curve $C$ imbedded in $S$ determines a complex line bundle as follows: Let $\left\{U_{\lambda}\right\}$ be a locally finite open covering of $S$ which admits a system of local coordinates $\left(z_{\lambda}, R_{\lambda}\right)$ on $U_{\lambda}$. In the case where $C \cap U_{\lambda} \neq \phi$ we assume that $R_{\lambda}$ is a defining equation of $C$. Hence letting

$$
R_{\lambda}=f_{\lambda \mu} R_{\mu} \quad \text { on } \quad U_{\lambda} \cap U_{\mu},
$$

we obtain a 1-cocycle $\left\{f_{\lambda \mu}\right\}$ which determines a complex line bundle [C]. The normal bundle $N_{C}$ is defined by $\left.[C]\right|_{c}$. A metric $\left\{a_{\lambda}\right\}$ of $[C]$ determines a $C^{\infty}$-function $F$ and a neighborhood system $V_{\varepsilon}$ of $C$ as follows:

$$
\begin{aligned}
F & =a_{\lambda}\left|R_{\lambda}\right|^{2}, \\
V_{\varepsilon} & =\{p \in S: F(p)<\varepsilon\} \quad \text { for small } \varepsilon>0 .
\end{aligned}
$$

We make the following definition: 
Definition (1.1). $C$ has a regularly half pseudoconvex neighborhood system $V_{\varepsilon}=\{F<\varepsilon\}$ if there exist a metric $\left\{a_{\lambda}\right\}$ of $[C]$ and a system of local coordinates $\left(z_{\lambda}, R_{\lambda}\right)$ on $U_{\lambda}$ such that

$$
\partial \bar{\partial} F=\beta_{\lambda} d R_{\lambda} \wedge d \bar{R}_{\lambda} \quad \text { where } \quad \beta_{\lambda}>0 .
$$

By $\mathcal{O}\left(V_{\varepsilon}\right)$ (resp. $\left.\mathscr{M}\left(V_{\varepsilon}\right)\right)$ we indicate the algebra (resp. field) of holomorphic (resp. meromorphic) functions on $V_{\varepsilon}$. In what follows functions are assumed to be of $C^{\infty}$-class. A domain $D$ with $D \Subset S$ is called (strongly-)pseudoconvex if at any point $p \in \partial D$ there exist a neighborhood $U$ and a (strongly-)pseudoconvex function $\varphi$ on $U$ satisfying $D \cap U$ $=\{\varphi<0\}$. By $s$-pseudoconvex domains (resp. functions) we mean stronglypseudoconvex domains (resp. functions). Similarly (s-)pseudoconcave domains are also defined.

\section{$\S 2$. The Necessity Part of Main Theorem}

We fix a metric $\left\{a_{\lambda}\right\}$ of $[C]$ and consider $V_{\varepsilon}$ for $0<\varepsilon \ll 1$ as a small neighborhood of $C$. Assume that $\mathcal{O}\left(V_{\varepsilon}\right) \nsubseteq \mathbf{C}$ holds.

At first we consider the following case:

$(\alpha)$ Any non-constant holomorphic function $f$ wihch vanishes on $C$ satisfies

$$
\{f=0\} \supsetneqq C \text {. }
$$

In this case we have the following:

Proposition (2.1)。 $N_{C}$ is negative.

Proof. Take such a function $f$. Then $\{f=0\}=C \cup \widetilde{C}$ where $\tilde{C}$ is a (possibly reducible) curve. Let $k$ be the order of $f$ at $C$. Then $\varphi_{\lambda}=f / R_{\lambda}^{k}$ determines a holomorphic section of $[C]^{-k}$ on $V_{\varepsilon}$. Restricting $\left\{\varphi_{\lambda}\right\}$ to $C$ we get a section of $N_{C}^{-k}$, which induces a positive divisor on C. The following Proposition is well known (see, H. Grauert [1] and H. Rossi [8]):

Proposition (2.2). (1) If $N_{C}$ is negative, then $C$ has an s-pseudoconvex neighborhood system, and so $C$ is an exceptional curve in the 
sense of H. Grauert [1]. (2) If $N_{C}$ is positive, then $C$ has an $s$ pseudoconcave neighborhood system.

Proof. We prove the first part of (1). The proof (2) is similar and may be omitted. By definition there exists a metric $\left\{\underline{a}_{\lambda}\right\}$ of $N_{\boldsymbol{C}}$ satisfying $\frac{\partial^{2} \log a_{\lambda}}{\partial z_{\lambda}^{1} \partial \bar{z}_{\lambda}^{1}}>0$ on $C \cap U_{\lambda}$. Extending this metric on $V_{\varepsilon}$, we have a metric $\left\{a_{\lambda}\right\}$ of $[C]$. Set $\tilde{a}_{\lambda}=a_{\lambda} \mathrm{e}^{\chi(F)}$ with a convex increasing function $\chi$. Choosing $\chi^{\prime}(0)$ sufficiently large and replacing $\varepsilon$ by a smaller constant $\varepsilon$, we get a negative metric $\left\{a_{\lambda}\right\}$ on $V_{\varepsilon}$. Let $\tilde{F}=\tilde{a}_{\lambda}\left|R_{\lambda}\right|^{2}$ and $\tilde{V}_{\varepsilon}=\{\tilde{F}<\varepsilon\}$. Then we obtain an s-pseudoconvex neighborhood system $\tilde{V}_{\varepsilon}$. To prove the second part of (1), we shall construct a contracting mapping as follows: Referring to $[C]^{-1}>0$ and the completeness of $\psi=1 /\left(1-\frac{F}{\varepsilon}\right)$ on $\tilde{V}_{\varepsilon}$, by S. Nakano's theorem (see, S. Nakano [6], p. 169, Theorem 1) we have

$$
H^{q}\left(\tilde{V}_{\varepsilon}, \mathcal{O}\left([C]^{-m}\right)\right)=0 \quad \text { for } \quad m \geqq m_{0} \quad \text { and } \quad q \geqq 1 \text {, }
$$

where $m_{0}$ is a certain positive integer. Then following $\mathrm{K}$. Kodaira [5] there exists a positive integer $m_{0}$ such that for any integer $m$ with $m \geqq$ $m_{0}$, (1) for any pair of points $p, q$ in $\tilde{V}_{\varepsilon}$ there exists a section $\varphi \in H^{0}\left(\tilde{V}_{\varepsilon}\right.$, $\mathcal{O}\left([C]^{-m}\right)$ ) satisfying $\varphi(p) \neq 0$ and $\varphi(q)=0$ and (2) for any point $p \in \tilde{V}_{\varepsilon}$ $\cap U_{\lambda}$ and constants $c_{1}, c_{2}$ and $c_{3}$, there exists a section $\psi \in H^{0}\left(\tilde{V}_{\varepsilon}\right.$, $\left.\mathcal{O}\left([C]^{-m}\right)\right)$ with

$$
\psi_{\lambda}(p)=c_{1}, \frac{\partial \psi_{\lambda}}{\partial z_{\lambda}}(p)=c_{2} \quad \text { and } \quad \frac{\partial \psi_{\lambda}}{\partial R_{\lambda}}(p)=c_{3}
$$

Since $\left\{\varphi_{\lambda}\right\} \in H^{0}\left(\tilde{V}_{\varepsilon}, \mathcal{O}\left([C]^{-m}\right)\right)$ determines a holomorphic function $f=$ $\varphi_{\lambda} R_{\lambda}^{m}$ on $\tilde{V}_{\varepsilon}$, from (1) and (2) we can derive (1') for any pair of points $p, q$ in $\tilde{V}_{\varepsilon}-C$ there exists a function $f$ such that $f(p) \neq 0, f(q)=0$ and $f=0$ on $C$ and $\left(2^{\prime}\right)$ for any point $p \in C$ there exists $f_{1}$ and $f_{2}$ such that $\frac{\partial\left(f_{1}, f_{2}\right)}{\partial\left(z_{\lambda}, R_{\lambda}\right)} \neq 0$ on $U(p)-C$ where $U(p)$ is a small neighborhood of p. By $\left(1^{\prime}\right)$ we have a system of global holomorphic functions $f_{1}, f_{2}, \ldots, f_{n}$ such that $\bigcap_{j=1}^{n}\left\{f_{j}=0\right\}=C$. Moreover, using (2') we can find $f_{n+1}, f_{n+2}, \ldots$, $f_{r}$ such that $\Psi_{r}=\left(f_{1}, f_{2}, \ldots, f_{r}\right): \widetilde{V}_{\varepsilon} \rightarrow \mathbb{C}^{r}$ is a proper mapping on $V^{*}$ $=\Psi_{r}^{-1}\left(D_{\delta}\right)$ which is also maximal rank on $V_{\delta}^{*}-C$, where $D_{\delta}$ is a small polydisc at 0 . Adding more functions $f_{r+1}, f_{r+2}, \ldots, f_{m}$ and replacing 
$\Psi_{r}$ by $\Psi_{m}=\left(f_{1}, f_{2}, \ldots, f_{m}\right)$, we may assume that in view of $\left(1^{\prime}\right)$ every fibre is connected. Thus we obtain a desired contracting mapping.

Consequently, in the case of $(\alpha)$, putting $\Psi_{m}\left(V_{\delta}^{*}\right)=\underline{V}_{\delta}^{*}$, we have a 2-dimensional normal Stein space $\underline{V}_{\delta}^{*}$ satisfying $\mathcal{O}\left(V_{\delta}^{*}\right)=\mathcal{O}\left(V_{\delta}^{*}\right)$.

Remark. Conversely if $C$ has an $s$-pseudoconvex neighborhood system, then $N_{C}$ is negative (see, H. Grauert [1], p. 355, Satz 9). But in the case of finite order, $C$ may have an s-pseudoconcave neighborhood system (see, Theorem 3).

The remaining case is the following:

$(\beta)$ There exists a holomorphic function $f$ on $V_{\varepsilon}$ satisfying $\{f=0\}$ $=k C$ with some positive integer $k$.

Replacing $V_{\varepsilon}$ by $V_{\delta}^{*}=\left\{p \in V_{\varepsilon}:|f(p)|<\delta\right\}$ with a small constant $\delta$, we may assume that $g=\left.f\right|_{V_{\delta}^{*}}$ is a proper mapping onto a disk $D_{\delta}$. Using Stein factorization of $g$ we obtain $\mathcal{O}\left(V_{\partial}^{*}\right) \cong \mathcal{O}(\mathbf{D})$ where $\mathbf{D}$ denotes the unit disk. As in the case $(\alpha)$, set $\left\{\varphi_{\lambda}=f / R_{\lambda}^{k}\right\}$. Then $\left\{\varphi_{\lambda}\right\} \in H^{0}\left(V_{\delta}^{*}\right.$, $\left.\mathcal{O}\left([C]^{-m}\right)\right)$. In this case $\left\{\varphi_{\lambda}\right\}$ vanishes nowhere and so $N_{C}^{-k}$ is analytically trivial. Any branch of $\varphi_{\lambda^{\frac{1}{k}}}$ defines a function on $U_{\lambda}$, which is also denoted by the same letter. Defining $z_{\lambda}^{*}=z_{\lambda}$ and $R_{\lambda}^{*}=\varphi_{\lambda^{\frac{1}{k}}} R_{\lambda}$, we have another system of local coordinates $\left(z_{\lambda}^{*}, R_{k}^{*}\right)$ on $U_{\lambda}$. Moreover,

$$
\partial \bar{\partial} F=d R_{\lambda}^{*} \wedge d \bar{R}_{\lambda}^{*} \quad \text { where } \quad F=\left|R_{\lambda}^{*}\right|^{2} .
$$

We remark that in (2.3), $\left\{a_{\lambda}=1\right\}$ can be chosen as a metric of $[C]$ and so the necessity part of Main Theorem is hereby proved.

Corollary (2.4). If there exists a curve $C$ on $S$ such that $N_{C}$ is positive or $N_{C}$ is of infinite order, then $\mathcal{O}(S) \cong \mathbb{C}$.

\section{§3. Holomorphic Foliations and Regularly Half Pseudoconvexity}

Let $\mathscr{D}$ be a 1-dimensional holomorphic differential system on $S$. $\omega=0$ denotes its praffian equation. In what follows we assume that $\mathscr{D}$ is completely integrable. Then for any $p \in S$ there exists one and only one maximal integral manifold $\mathscr{S}_{p}$ through $p$, which is called a leaf of $\mathscr{D}$. We call $\mathscr{F}=\left\{\mathscr{S}_{p}\right\}$ a holomorphic foliation. Given two foliations $\mathscr{F}_{1}$ and $\mathscr{F}_{2}$ on $S, \mathscr{F}_{1}$ is said to be equivalent to $\mathscr{F}_{2}$ when 
their leaves coincide completely. $\mathscr{F}$ is called a closed foliation if every leaf is a closed submanifold in $S$. We say that $\mathscr{F}$ is globally integrable when $\mathscr{F}$ is equivalent to a foliation defined by a global holomorphic function on $S$.

Theorem 1. If $C$ has a regularly half pseudoconvex neighborhood system, then there exists a holomorphic foliation on $V_{\varepsilon}$ for a small $\varepsilon$.

Proof. From (1.1)

$$
\begin{gathered}
F=a_{\lambda}\left|R_{\lambda}\right|^{2}, \\
\partial_{z_{\lambda}} \bar{\partial}_{z_{\lambda}} F=0, \\
\bar{\partial}_{R_{\lambda}} \partial_{z_{\lambda}} F=0 \text { and } \bar{\partial}_{z_{\lambda}} \partial_{R_{\lambda}} F=0 .
\end{gathered}
$$

By (3.2) we have

$$
F=a_{0}\left(R_{\lambda}\right)+\sum_{k=1}^{\infty} a_{k}\left(R_{\lambda}\right) z_{\lambda}^{k}+\sum_{k=1}^{\infty} \bar{a}_{k}\left(R_{\lambda}\right) \bar{z}_{\lambda}^{k} .
$$

Therefore

$$
\partial_{z_{\lambda}} F=\sum_{k=1}^{\infty} k a_{k}\left(R_{\lambda}\right) z_{\lambda}^{k-1}
$$

which is a holomorphic function of $z_{\lambda}$ when $R_{\lambda}$ is fixed. On the other hand (3.3) implies that $\partial_{z_{\lambda}} F$ is also a holomorphic function of $R_{\lambda}$ when $z_{\lambda}$ is fixed. Hence by Hartogs' theorem it is a holomorphic function of $z_{\lambda}$ and $R_{\lambda}$. Thus

$$
a_{k}\left(R_{\lambda}\right)=\sum_{j=0}^{\infty} a_{k, j} R_{\lambda}^{j} \quad(k=1,2,3, \ldots)
$$

Put $z_{\lambda}=0$ in (3.1) we have $a_{0}\left(R_{\lambda}\right)=a_{\lambda}\left(0, R_{\lambda}\right)\left|R_{\lambda}\right|^{2}$. Put $R_{\lambda}=0$ in (3.4) and taking (3.5) into account, we see that $a_{k, 0}=0(k=1,2, \ldots)$. This shows that $F$ and the first and the second terms of the right hand of (3.4) can be divided by $R_{\lambda}$ and its quotient is a $C^{\infty}$-function on $U_{\lambda}$. Thus $\Sigma \bar{a}_{k}\left(R_{\lambda}\right) \bar{z}_{\lambda}^{k}$ must be also divided by $R_{\lambda}$ and its quotient also must be a $C^{\infty}$-function on $U_{\lambda}$. Consequently we obtain that $a_{k, j}=0(k=1$, $2, \ldots$ and $j=0,1, \ldots)$, which imply 


$$
F=a_{\lambda}\left(0, R_{\lambda}\right)\left|R_{\lambda}\right|^{2} .
$$

This yields

$$
\frac{\partial F}{\partial z_{\mu}}=\frac{\partial F}{\partial R_{\lambda}} \frac{\partial R_{\lambda}}{\partial z_{\mu}}
$$

and

$$
\frac{\partial^{2} F}{\partial z_{\mu} \partial \bar{z}_{\mu}}=\frac{\partial^{2} F}{\partial R_{\lambda} \partial \bar{R}_{\lambda}}\left|\frac{\partial R_{\lambda}}{\partial z_{\mu}}\right|^{2} \quad \text { on } \quad U_{\lambda} \cap U_{\mu}
$$

By the assumption we have $\frac{\partial R_{\lambda}}{\partial z_{\mu}}=0$. Hence

$$
R_{\lambda}=f_{\lambda \mu}\left(R_{\mu}\right) R_{\mu} \quad \text { on } \quad U_{\lambda} \cap U_{\mu}
$$

Therefore $d R_{\lambda}=\xi_{\lambda \mu} d R_{\mu}$ on $U_{\lambda} \cap U_{\mu}$, where $\xi_{\lambda \mu}=f_{\lambda \mu}+\frac{\partial f_{\lambda \mu}}{\partial R_{\mu}} R_{\mu}$. Making $\varepsilon$ smaller, we may assume that $\xi_{\lambda \mu} \neq 0$ on $V_{\varepsilon}$. Then we have a holomorphic foliation $\mathscr{F}$ on $V_{\varepsilon}$ by the following equation:

$$
\omega=0 \quad \text { where } \omega=d R_{\lambda},
$$

which is denoted by $\mathscr{F}_{F}$.

Corollary. Under the assumption in Theorem $1, F$ and $\left\{f_{\lambda \mu}\right\}$ can be expressed as follows:

$$
\begin{aligned}
& F=a_{\lambda}\left(R_{\lambda}\right)\left|R_{\lambda}\right|^{2}, \\
& R_{\lambda}=f_{\lambda \mu}\left(R_{\mu}\right) \cdot R_{\mu} .
\end{aligned}
$$

Remark 1. The concept of regularly half pseudoconvexity can be generalized to a compact kähler manifold imbedded as a divisor in another complex manifold.

Remark 2. As for the degeneracy of holomorphic functions and the foliation structure, see A. T. Huckleberry and R. Nirenberg [4].

\section{§4. The Sufficiency Part of Main Theorem}

Given a curve $C$ with a regularly half pseudoconvex neighborhood, 
then (3.6) and (3.7) hold with respect to the coordinates $\left(z_{\lambda}, R_{\lambda}\right)$ on $U_{\lambda}$ given in (2.4) and the foliation $\mathscr{F}_{F}$ is obtained. Let $\left\{U_{\lambda}^{*}\right\}$ be a finite open covering of $V_{\varepsilon}$ defined by $U_{\lambda}^{*}=\left\{\left(z_{\lambda}, R_{\lambda}\right):\left|z_{\lambda}\right|<\rho, F<\varepsilon\right\}$ where $\rho$ is a positive constant. For each $\lambda$, we choose a point $p_{\lambda}^{(0)}=\left(z_{\lambda}^{(0)}, R_{\lambda}^{(0)}\right)$ $\in U_{\lambda}^{*}$ and define $D_{\lambda}^{(\varepsilon)}$ as follows: $D_{\lambda}^{(\varepsilon)}=V_{\varepsilon} \cap\left\{z_{\lambda}=z_{\lambda}^{(0)}\right\}$, which may be assumed to be a small disk. First from (3.6) and (3.7) we infer that if $F$ attains a value $c$ at a point in $\mathscr{S}$, then $F(p)=c$ for any point $p \in \mathscr{S}$ for each leaf $\mathscr{S} \in \mathscr{F}_{F}$. This implies that for any point $p \in V_{\varepsilon}$, the leaf through $p$ is completely contained in $V_{\varepsilon}$. Fix a point $p \in C$ $\cap U_{0}^{*}$ and consider a $C^{\infty}$-path $\gamma=\{\gamma(t): 0 \leqq t \leqq 1\}$ such that $\gamma(0)=p$ and $\gamma \subset C$. By $\gamma^{-1}$ we denote a path $\{\gamma(1-t): 0 \leqq t \leqq 1\}$. Now we take an analytic set $\left\{R_{0}=c_{0}\right\}$ in $V_{\varepsilon} \cap U_{0}^{*}$ for a constant $c_{0}$ and define a continuation of $\left\{R_{0}=c_{0}\right\}$ along $\gamma$ as follows: Take $t_{0}$ such that $\gamma_{0}=\{\gamma(t)$ : $\left.0 \leqq t<t_{0}\right\} \subset U_{0}^{*}$ and $\gamma\left(t_{0}\right) U \notin$ N $_{0}^{*}$. Choose $U_{1}^{*}$ with $t_{0} \in U_{1}^{*}$. Next choose $t_{1}$ and $U_{2}^{*}$ such that $\gamma_{1}=\left\{\gamma(t): t_{0} \leqq t<t_{1}\right\} \subset U_{1}^{*}, \gamma\left(t_{1}\right) \notin U_{1}^{*}$ and $\gamma\left(t_{1}\right) \in U_{2}^{*}$. By repeating this process we have $\left(\gamma_{0}, U_{0}^{*}\right),\left(\gamma_{1}, U_{1}^{*}\right), \ldots,\left(\gamma_{m}, U_{m}^{*}\right)$, where $\left.\gamma_{m}=\left\{\gamma(t): t_{m-1} \leqq t \leqq 1\right)\right\} \subset U_{m}^{*}$. For each $\gamma_{i}$ we define inductively an analytic set $\left\{R_{i}=c_{i}\right\}$ on $U_{1}^{*}$ by the condition

$$
c_{i}=f_{i, i-1}\left(c_{i-1}\right) c_{i-1} \quad(i=1,2, \ldots, m)
$$

Hence we have an analytic set $S\left(c_{0}\right)$ in $V=\bigcup_{i=1}^{m} U_{i}^{*}$ as follows:

$$
S\left(c_{0}\right)=\bigcup_{i=1}^{m}\left\{R_{i}=c_{i}\right\}
$$

We call $S\left(c_{0}\right)$ the continuation of $\left\{R_{0}=c_{0}\right\}$ along $\gamma$ in $V$. By the compatibility condition of $\left\{f_{\lambda \mu}\right\}, S\left(c_{0}\right)$ does not depend on the choices of $\left\{U_{i}^{*}\right\}$. As for the continuation the following holds:

Proposition (4.1). For any path $\gamma \subset C$, define $\theta_{m, 0}(\gamma): D_{0}(\varepsilon) \rightarrow D_{m}(\varepsilon)$ by $\theta_{m, 0}(\gamma)\left(c_{0}\right)=c_{m}$. Then $\theta_{m, 0}(\gamma)$ is a biholomorphic mapping and $\theta_{m, 0}(0)$ $=0$. Moreover, if $\gamma$ is homotopic to $\gamma^{\prime}$, then $\theta_{m, 0}(\gamma)=\theta_{m, 0}\left(\gamma^{\prime}\right)$.

The proof is easy.

Proposition (4.2). If $C$ has a regularly half pseudoconvex neighborhood, then there exists a metric $\left\{\tilde{a}_{\lambda}\right\}$ on $V_{\varepsilon}$ and a system of local 
coordinates $\left(\tilde{z}_{\lambda}, \widetilde{R}_{\lambda}\right)$ on $U_{\lambda}^{*}$ such that (1) $\tilde{R}_{\lambda}=\tilde{f}_{\lambda \mu} \widetilde{R}_{\mu}$ where $\left|\tilde{f}_{\lambda \mu}\right|=1$ and (2) $\tilde{F}=\tilde{a}_{\lambda}\left|R_{\lambda}\right|^{2}$ defines a regularly half pseudoconvex neighborhood $\tilde{V}_{\varepsilon}=\{F<\varepsilon\}$ of $C$.

Proof. We choose a path $\gamma_{\lambda} \subset C$ such that $\gamma_{\lambda}(0)=p_{0}^{(0)} \in U_{0}^{*}$ and $\gamma_{\lambda}(1)$ $=p_{\lambda}^{(0)} \in U_{\lambda}^{*}$. By (4.1) we have a biholomorphic mapping $\theta_{\lambda, 0}\left(\gamma_{\lambda}\right): D_{0}(\varepsilon)$ $\rightarrow D_{\lambda}(\varepsilon)$, whose inverse is denoted by $\varphi_{\lambda}$. We can define a new local coordinates $\left(\tilde{z}_{\lambda}, \widetilde{R}_{\lambda}\right)$ on $U_{\lambda}$ as follows:

$$
\tilde{z}_{\lambda}=z_{\lambda}, \quad \tilde{R}_{\lambda}=\varphi_{\lambda}\left(R_{\lambda}\right) .
$$

Now take any point $p \in U_{\lambda}^{*} \cap U_{\mu}^{*}$. Choosing a path $\delta_{\lambda}$ (resp. $\delta_{\mu}$ ) in $U_{\lambda}^{*}$ $\cap U_{\mu}^{*}$ such that $\delta_{\lambda}(0)=p$ and $\delta_{\lambda}(1)=p_{\lambda}^{(0)}\left(\operatorname{resp} . \delta_{\mu}(0)=p\right.$ and $\left.\delta_{\mu}(1)=p_{\mu}^{(0)}\right)$, we have a closed path $\gamma_{\lambda \mu}^{\prime}=\gamma_{\mu}^{-1} \circ \delta_{\mu} \circ \delta_{\lambda}^{-1} \circ \gamma_{\lambda}$. We replace $\gamma_{\lambda \mu}^{\prime}$ by a path $\gamma_{\lambda \mu}$ in $C$ which is homotopic to $\gamma_{\lambda \mu}^{\prime}$. We may assume that the homotopy class of $\gamma_{\lambda \mu}$ does not depend on the choices of $p$ and $\delta_{\lambda}, \delta_{\mu}$. By (4.1) $\theta_{0,0}\left(\gamma_{\lambda \mu}\right)$ is a biholomorphic automorphism of $D_{0}(\varepsilon)$, which we write as $\varphi_{\lambda \mu}$. Then with some constant $\alpha_{\lambda \mu}$ it can be written as

$$
\varphi_{\lambda \mu}\left(R_{0}\right)=\alpha_{\lambda \mu} \cdot R_{0} \quad \text { where } \quad\left|\alpha_{\lambda \mu}\right|=1
$$

Accordingly

$$
\widetilde{R}_{\lambda}(p)=\alpha_{\lambda \mu} \cdot \widetilde{R}_{\mu}(p) \quad \text { for } \quad p \in U_{\lambda} \cap U_{\mu} .
$$

We define $\left\{\phi_{\lambda}\left(R_{\lambda}\right)\right\}$ by $\varphi_{\lambda}\left(R_{\lambda}\right)=\phi_{\lambda}\left(R_{\lambda}\right) R_{\lambda}$. Then $\phi_{\lambda}\left(R_{\lambda}\right) \neq 0$ everywhere. In view of $\widetilde{R}_{\lambda}(p)=\varphi_{\lambda}\left(f_{\lambda \mu}\left(R_{\mu}\right) \cdot R_{\mu}(p)\right)$ for $p \in U_{\lambda} \cap U_{\mu}$, we obtain

$$
\phi_{\lambda}\left(f_{\lambda \mu}\left(R_{\mu}(p)\right) R_{\mu}(p)\right) \cdot f_{\lambda \mu}\left(R_{\mu}(p)\right)=\alpha_{\lambda \mu} \cdot R_{\mu}(p),
$$

which implies that $\left\{\alpha_{\lambda \mu}\right\}$ is equivalent to [C]. It is easily seen that $\tilde{a}_{\lambda}=a_{\lambda}\left|\phi_{\lambda}\right|^{2}$ satisfies the assertion (2) in (4.2).

Now we prove the sufficiency part of Main Theorem. If $N_{C}$ is negative, then $\mathcal{O}\left(V_{\varepsilon}\right) \nsubseteq \mathbf{C}$ by (2.2). If $C$ has a regularly half pseudoconvex neighborhood, by (4.2) it may be assumed that $\left|f_{\lambda \mu}\right|=1$. Since $N_{C}^{k}=1$, $\left\{f_{\lambda \mu}^{k}\right\}$ is analytically trivial on $C$. Hence there exists a system of nonvanishing holomorphic functions $\left\{\alpha_{\lambda}\right\}$ on $U_{\lambda} \cap C$ such that

$$
f_{\lambda \mu}^{k}=\alpha_{\mu} \cdot \alpha_{\lambda}^{-1}
$$


In view of $\left|f_{\lambda \mu}\right|=1$, we have a harmonic function $h=\left|\alpha_{\lambda}\right|$ on $C$. Then $\alpha_{\lambda}$ must be constant. Therefore (4.3) holds on $V_{\varepsilon}$. Recalling $f_{\lambda \mu}=R_{\lambda} / R_{\mu}$, we get a holomorphic function

$$
f=\alpha_{\lambda} R_{\lambda}^{k} \quad \text { on } \quad V_{\varepsilon} \text {. }
$$

We remark that $\{f=0\}=k C$.

\section{§5. Examples}

In this section we consider two examples due to H. Grauert [2] and $M$. Otuki [7] respectively.

Example 1. Let $C$ be a compact Riemann surface of genus $g(g \geqq 1)$ and let $E$ be a topologically trivial line bundle on $C$. $\pi$ denotes the natural projection of $E$. We cover $C$ by an open covering $\left\{V_{\lambda}\right\}$ such that $E \mid V_{\lambda}$ is analytically trivial. The local coordinate of $V_{\lambda}$ and the fiber coordinate of $\pi^{-1}\left(V_{\lambda}\right)$ are denoted by $z_{\lambda}$ and $\zeta_{\lambda}$ respectively. By a well known lemma we may assume

$$
\left|f_{\lambda \mu}\right|=1 \quad \text { on } \quad V_{\lambda} \cap V_{\mu} .
$$

So we have a tubular neighborhood system $T_{\varepsilon}$ of the zero section, $T_{\varepsilon}=\left\{\left(z_{\lambda}, \zeta_{\lambda}\right):\left|\zeta_{\lambda}\right|^{2}<\varepsilon\right\}$. We infer that $T_{\varepsilon}$ is always a regularly half pseudoconvex neighborhood system of $C$. We define a differential system $\mathscr{D}$ by

$$
d \zeta_{\lambda}=0 \quad \text { on } \quad \pi^{-1}\left(V_{\lambda}\right) \cap T_{\varepsilon}
$$

Since $\left|f_{\lambda \mu}\right|=1, \mathscr{D}$ is well defined on $T_{\varepsilon}$ and a holomorphic foliation $\mathscr{F}$ is obtained on $T_{\varepsilon}$.

Theorem 2. (1) If $E$ is of finite order, then $\mathscr{F}$ is globally integrable and $\mathcal{O}\left(T_{\varepsilon}\right) \cong \mathcal{O}(\mathbf{D})$ where $\mathbf{D}$ is the unit disk. (2) If $E$ is of infinite order, $\mathscr{F}$ can never be a closed foliation and $\mathcal{O}\left(T_{\varepsilon}\right) \cong \mathbf{C}$.

Proof. By regularly half pseudoconveity of $T_{\varepsilon}$, (1) is a direct consequence of Main Theorem. For the proof of (2) assume that $\mathscr{F}$ would be a closed foliation. Then a leaf $\mathscr{S}_{p}$ through $p \in T_{\varepsilon} \cap \pi^{-1}\left(V_{\lambda}\right)$ 
can be expressed as $\left\{\zeta_{\lambda}=c_{\lambda}\right\}$ on $T_{\varepsilon} \cap \pi^{-1}\left(V_{\lambda}\right)$, where $c_{\lambda}=\zeta_{\lambda}(p)$. Since $\left|f_{\lambda \mu}\right|=1, \mathscr{S}_{p}$ has finite intersection points with any fiber $\pi^{-1}(p), p \in C$. The connected components of $\mathscr{S}_{p}$ on $\pi^{-1}\left(V_{\lambda}\right) \cap T_{\varepsilon}$ are denoted by $\left\{A_{\mu}^{(i)}\right\}$ $(i=1,2, \ldots, k)$ where $A_{\mu}^{(i)}=\left\{\zeta_{\mu}=c_{\mu}^{(i)}\right\}$. Note that $k$ does not depend on $\mu$. For each $\mu$ we prepare $k$ copies of $\pi^{-1}\left(V_{\lambda}\right) \cap T_{\varepsilon}$ which are denoted by $W_{\mu}^{(i)}(i=1,2, \ldots, k)$ whose local coordinates are denoted by $\left(z_{\lambda}, \zeta_{\mu}^{(i)}\right)$. We identify a point $p_{\lambda}^{(i)}=\left(z_{\lambda}, \zeta_{\lambda}^{(i)}\right) \in W_{\lambda}^{(i)}$ with a point $p_{\mu}^{(j)}$ $=\left(z_{\mu}, \zeta_{\mu}^{(j)}\right) \in W_{\mu}^{(j)}$ by the following conditions: (1) $\pi\left(p_{\lambda}^{(i)}\right)=\pi\left(p_{\mu}^{(j)}\right)$ (2) $c_{\lambda}^{(i)}=f_{\lambda \mu} c_{\mu}^{(j)}$ and (3) $\zeta_{\lambda}^{(i)}=f_{\lambda \mu} \zeta_{\mu}^{(j)}$. Then we have a $k$-fold unramified covering manifold $\tilde{T}_{\varepsilon}$. The natural projection is denoted by $\omega$. By construction there exists an unramified covering $\tilde{C}$ over $C, \omega^{\prime}: \widetilde{C} \rightarrow C$, such that $\widetilde{T}_{\varepsilon}$ coincides with a tubular neighborhood of $\omega^{\prime *}(E)$. Then $\omega^{\prime *}(E)$ admits a trivial section, which contradicts the assumption of infinite order. For the proof of the second part of (2) we consider $f \in \mathcal{O}\left(T_{\varepsilon}\right)$. Since $\left|f_{\lambda \mu}\right|=1, f$ can be expressed as follows:

$$
f=\sum_{m=0}^{\infty} a_{\lambda}^{(m)} \zeta_{\lambda}^{m} \quad \text { on } \quad T_{\varepsilon} \cap \pi^{-1}\left(V_{\lambda}\right)
$$

where the $a_{\lambda}^{(m)}$ are constants on $V_{\lambda}$. If $f$ were non-constant, $\{f=c\}$ would be an analytic set in $T_{\varepsilon}$ whose irreducible component could be expressed as $\left\{\zeta_{\mu}=b\right\}$ with some constant $b$ on $\pi^{-1}\left(V_{\mu}\right)$. This contradicts the non-closedness of $\mathscr{F}$. Thus we obtain $\mathcal{O}\left(T_{\varepsilon}\right) \cong \mathbf{C}$. This is an alternative proof of the theorem of $\mathrm{H}$. Grauert [2]:

Remark. In Example 1, $C$ has always a regularly half pseudoconvex neighborhood system $T_{\varepsilon}$. This implies that regularly half pseudoconvexity is not a sufficient condition for the existence problem.

Example 2. Hopf surfaces are defined in the following manner: Let $\mathbf{C}^{2}=\left\{\left(z_{1}, z_{2}\right)\right\}$ and $\mathbf{W}=\mathbb{C}^{2}-\{(0,0)\}$ and consider a holomorphic automorphism of $\mathbf{W}$

$$
g:\left(z_{1}, z_{2}\right) \longrightarrow\left(\alpha_{1} z_{1}+\lambda z_{2}^{m}, \alpha_{2} z_{2}\right)
$$

where $\alpha_{1}, \alpha_{2}$ and $\lambda$ are constants and $m$ is a positive integer with the conditions $\left(\alpha_{1}-\alpha_{2}^{m}\right) \lambda=0$ and $0<\left|\alpha_{1}\right| \leqq\left|\alpha_{2}\right|<1$. Then $G=\left\{g^{n}: n \in \mathbb{Z}\right\}$ acts on $\mathbf{W}$ properly discontinuously, which determines a compact surface 
$S=\mathbf{W} / G$. The surface thus obtained is called a Hopf surface. $\pi$ denotes the natural projection, $\pi: \mathbf{W} \rightarrow S$. The curve $\left\{z_{2}=0\right\}$ is always invariant by $G$, which defines a non-singular elliptic curve $C$ on $S$. Furthermore, in the case where $\lambda=0,\left\{z_{1}=0\right\}$ also induces another elliptic curve $C_{1}$. The following is essetially due to M. Otuki [5].

Theorem 3. (1) If $\lambda=0$, then $C$ (or $C_{1}$ ) has a regularly half pseudoconvex neighborhood system $V_{\varepsilon}$. Moreover, (i) if $\alpha_{1}^{k}=\alpha_{2}^{j}$ with some positive integer $k$ and $j$, then $\mathcal{O}\left(V_{\varepsilon}\right) \cong \mathcal{O}(\mathbf{D})$ and (ii) if $\alpha_{1}^{k} \neq \alpha_{2}^{j}$ for any pair of non-zero integers $k$ and $j$, then $\mathcal{O}\left(V_{\varepsilon}\right) \cong \mathbf{C}$. (2) If $\lambda \neq 0, C$ has an s-pseudoconcave neighborhood system and $N_{C}^{m}=1$. Moreover, $\mathcal{O}\left(V_{\varepsilon}\right) \cong \mathbf{C}$.

Proof. We prove (1) only in the case of $C$. We write $\left|\alpha_{1}\right|=\rho$, $\left|\alpha_{2}\right|=\sigma$ and define $\tau$ by $\sigma=\rho^{\tau}$. By (5.1) $F=\left|z_{2}\right|^{2} /\left|z_{1}^{\tau}\right|^{2}$ is invariant by G. So $F$ is a function on $S-C_{1}$ and $V_{\varepsilon}=\{F<\varepsilon\}$ is a neighborhood system of $C$. Define a differential system $\mathscr{D}$ on $W_{\varepsilon}=\pi^{-1}\left(V_{\varepsilon}\right)$ by

$$
\omega=0, \quad \omega=d\left(z_{2} / z_{1}^{\tau}\right)
$$

where one of the branchs of $z_{1}^{\tau}$ is fixed. Then $\mathscr{D}$ is completely integrable and induces a foliation $\widetilde{\mathscr{F}}$ on $W_{\varepsilon}$. Every leaf is nothing but the analytic continuation of the analytic set $\left\{z_{2}=z_{1}^{\tau} c\right\}$ for $|c|^{2}<\varepsilon$, which is denoted by $\mathscr{S}_{c}$. Since $g^{*} \omega=h \omega$ where $h=\alpha_{2} / \alpha_{1}^{\tau}$, we get a foliation $\mathscr{F}=\left\{\pi\left(\mathscr{S}_{c}\right):|c|^{2}<\varepsilon\right\}$ on $V_{\varepsilon}$ from $\widetilde{\mathscr{F}}$. Note that $\pi\left(\mathscr{S}_{c}\right)$ has no intersection points with $\partial V_{\varepsilon}$. In the case of (i) $\tau=j / k$ holds. Then we have a global holomorphic function $f=\left(z_{2} / z_{1}^{\tau}\right)^{k}$ on $V_{\varepsilon}$. The foliation defined by $f$ is equivalent to $\mathscr{F}$. By the Stein factorization, we obtain $\mathcal{O}\left(V_{\varepsilon}\right)$ $\cong \mathcal{O}(\mathbf{D})$. In the case of (ii), $\tau$ is irrational. So we have $\overline{\pi\left(\mathscr{S}_{c}\right)}=\left\{F=|c|^{2}\right\}$, where $\overline{\pi\left(\mathscr{S}_{c}\right)}$ denotes the closure of $\pi\left(\mathscr{S}_{c}\right)$ in $S$. Take a holomorphic function $f$ on $V_{\varepsilon}$, then $f$ must be bounded on $\pi\left(\mathscr{S}_{c}\right)$. Since the universal covering surface of $\pi\left(\mathscr{S}_{c}\right)$ is isomorphic to C, we see that $f$ is constant on $\pi\left(\mathscr{S}_{c}\right)$. Hence by the theorem of identity we see that $f$ is constant on $V_{\varepsilon}$. To prove (2), it is sufficient to show

$$
S^{*}=\mathrm{C}^{*} \times \mathrm{C}^{*} \quad \text { where } \quad S^{*}=S-C
$$


Set $\mathbb{C}^{2}=\left\{\left(Z_{1}, Z_{2}\right)\right\}, D=\mathbb{C}^{2}-\left\{Z_{2}=0\right\}$ and define $\Phi: D \rightarrow \mathbb{W}-\left\{z_{2}=0\right\}$ by $\Phi\left(Z_{1}, Z_{2}\right)=\left(Z_{1} Z_{2}^{m}, Z_{2}\right)$. Then $\Phi$ is a biholomorphic mapping. With this identification $g$ can be expressed as $Z_{1}^{\prime}=Z_{1}+\lambda^{\prime}, Z_{2}^{\prime}=\alpha_{2} Z_{2}$ where $\lambda^{\prime}=\lambda / \alpha_{2}^{m}$. The universal covering manifold of $D$ is isomorphic to $\mathbb{C}^{2}$ $=(u, v)$ and the covering projection is written as $\omega:(u, v) \rightarrow\left(Z_{1}, Z_{2}\right)$ $=\left(u, \mathrm{e}^{v}\right)$. Therefore its covering transformation group is generated by

$$
g^{(1)}:\left\{\begin{array}{l}
u^{\prime}=u \\
v^{\prime}=v+2 \pi \sqrt{-1}
\end{array}\right.
$$

Also $g^{(2)}=\omega^{*}(g)$ can be written as follows:

$$
g^{(2)}:\left\{\begin{array}{l}
u^{\prime}=u+\lambda \\
v^{\prime}=v+\mu, \mu=\log \alpha_{2} .
\end{array}\right.
$$

Using a suitable linier transform in $\mathbf{C}^{2}$ we may assume that $g^{(1)}$ and $g^{(2)}$ are in the following form:

$$
g^{(1)}:\left\{\begin{array}{l}
u^{\prime}=u \\
v^{\prime}=v+a
\end{array} \quad(a \neq 0), \quad g^{(2)}:\left\{\begin{array}{l}
u^{\prime}=u+b \\
v^{\prime}=v
\end{array} \quad(b \neq 0) .\right.\right.
$$

Set $G^{(1)}=\left\{g^{(1)^{n}}: n \in \mathbb{Z}\right\}$ and $G^{(2)}=\left\{g^{(2)^{n}}: n \in \mathbf{Z}\right\}$, then

$$
S^{*}=\left[\mathbf{C}^{2} / G^{(1)}\right] / G^{(2)} \cong \mathbf{C}^{*} \times \mathbf{C}^{*} .
$$

$N_{C}^{m}=1$ can be proved as follows: $\psi=z_{2}^{-(m+1)} d z_{1} \wedge d z_{2}$ is a meromorphic 2-form on $\mathbf{W}$ which is invariant by $G$. So $\psi$ can be seen as a form on $S$. This implies that the canonical line bundle $K_{S}$ of $S$ can be written as follows:

$$
K_{S}=[C]^{-(m+1)}
$$

Referring to $K_{C}=1$, we obtain $N_{C}^{m}=1$ by the adjunction formula.

Remark 1. The finite order condition is not sufficient for the existence problem.

Remark 2. For another example of a compact curve $C$ such that (1) $N_{C}$ is of finite order and (2) $C$ has a s-pseudoconcave neighborhood system, see R. Hartshorne [3], Example 3.2, p. 232. 


\section{§6. Conclusions and Remarks}

By the discussions above we obtain

(1) if $N_{C}$ is negative, $C$ has an s-pseudoconvex neighborhood system $V_{\varepsilon}$ and there exists a 2-dimensional normal Stein space $V_{\varepsilon}^{*}$ satifying $\mathcal{O}\left(V_{\varepsilon}\right) \cong \mathcal{O}\left(V_{\varepsilon}^{*}\right)$ by $(2.2)$,

(2) if $N_{C}$ is positive, by (2.2) $C$ has an s-pseudoconcave neighborhood system and $\mathcal{O}\left(V_{\varepsilon}\right) \cong \mathbf{C}$ by $(2.5)$,

(3) in the case where $N_{C}$ is topologically trivial,

(i) if $N_{C}$ is of finite order, $\mathcal{O}\left(V_{\varepsilon}\right) \cong \mathcal{O}(\mathbf{D})$ if and only if $C$ has a regularly half pseudoconvex neighborhood system. Moreover, Theorem 3 shows that there exists a $C \subset S$ having an $s$-pseudoconcave neighborhood system even if $N_{C}$ satisfies the condition of finite order.

(ii) if $N_{C}$ is of infinite order, then $\mathcal{O}\left(V_{\varepsilon}\right) \cong \mathbf{C}$ by (2.5). Theorem 2 shows that there exists a curve $C \subset S$ having a regularly half pseudoconvex neighborhood system even if $N_{C}$ satisfies the condition of infinite order.

We summarize these results in the following Table:

Table

\begin{tabular}{|c|c|c|c|}
\hline \multicolumn{2}{|c|}{ normal bundle } & $\begin{array}{l}\text { pseudoconvexity of a } \\
\text { neighborhood system } V_{\varepsilon}\end{array}$ & $\begin{array}{l}\text { the Remmert } \\
\text { reduction of } V_{\varepsilon}\end{array}$ \\
\hline \multicolumn{2}{|c|}{ negative } & $s$-pseudoconvex & $\begin{array}{l}\text { 2-dimensional } \\
\text { normal Stein space }\end{array}$ \\
\hline \multicolumn{2}{|c|}{ positive } & $s$-pseudoconcave & $p$ \\
\hline \multirow{3}{*}{$\begin{array}{l}\text { topologically } \\
\text { trivial }\end{array}$} & \multirow{2}{*}{$\begin{array}{l}\text { finite } \\
\text { order }\end{array}$} & regularly half pseudoconvex & $\mathbf{D}$ \\
\hline & & $\begin{array}{c}\text { otherwise ( } s \text {-pseudoconcave } \\
\text { cases may occur) }\end{array}$ & $p$ \\
\hline & $\begin{array}{l}\text { infinite } \\
\text { order }\end{array}$ & $\begin{array}{l}\text { (regularly half pseudoconvex } \\
\text { cases may occur) }\end{array}$ & $p$ \\
\hline
\end{tabular}

Remark 1. When a compact curve $C$ with $N_{C}>0$ is imbedded in a 2-dimensional compact compex manifold $S, S$ must be a projective algebraic manifold. Moreover, for any neighborhood $V$ of $C, \mathscr{M}(V)$ $\cong \mathscr{M}(S)$. 
Remark 2. Let $C$ be a compact curve which has a pseudoconvex neighborhood system $V_{\rho}=\{F<\rho\}$ where $F$ is a certain pseudoconvex function on a neighborhood of $C$. Moreover, suppose that $C$ is of infinite order. Then $\mathcal{O}\left(V_{\rho}\right) \cong \mathbf{C}$, but there exists many meromorphic functions on $V_{\rho}$ for a small $\rho$. In fact, making $\rho$ smaller, we may assume that there exists a divisor $\tilde{C}$ which is transversal with $C$. In the similar manner as in $(2.2)$ we see that $[\widetilde{C}]>0$ for a small constant $\rho$. Using Nakano's theorem [4], we see that there exists a positive integer $n_{0}$ satisfying $H^{q}\left(V_{\rho}, \mathcal{O}\left([\widetilde{C}]^{n}\right)\right)=0$ for $q \geqq 1$ and $n \geqq n_{0}$. Then $V_{\rho}$ can be imbedded by sections of $[\tilde{C}]^{n}$ in a projective space. This shows that $V_{\rho}$ is meromorphically separable.

Remark 3. In the case of a non-singular non-compact curve imbedded in $S$, it can be proved that it has always a Stein neighborhood system by using H. Grauert's Lemma (see, H. Grauert [1], p. 340, Satz 5).

\section{References}

[1] Grauert, H., Über Modifikationen und exzeptionelle analyishe Mengen, Math. Ann., 146 (1962), 331-368.

[2] - Bemerkenswcrte pseudokonvexe Mannigfaltigkeiten, Math. Zeit., 81 (1965), 377-391.

[3] Hartshorne, R., Ample subvarieties of algebraic varieties, Lecture notes in Mathmatics, 156, Berlin-Heidelberg-New York, Springer (1970).

[4] Huckleberry, A. T. and Nirenberg, R., On k-pseudoflat complex spaces, Math. Ann., 200 (1973), 1-10.

[5] Kodaira, K., On KÄHLER varieties of restricted type (an intrinsic characterisation of algebraic varieties), Ann. Math., 60 (1954), 28-48.

[6] Nakano, S., Vanishing theorems for weakly 1-complete manifolds, "Number theory, Commutative algebra and Algebraic geometry", Kinokuniya, (1973), 169-179.

[7] Otuki, M., On iterations in $\mathbf{G}^{2}$, Master Thesis submitted to University of Tokyo, (1972).

[8] Rossi, H., Attaching analytic spaces to an analytic space along a pseudoconcave boundary, Proceedings of the conference on complex analysis Minneapolis 1964, Springer (1965). 
